\title{
High-energy band structure of gold
}

\section{Christensen, N. Egede}

\section{Published in:}

Physical Review B

Link to article, DOI:

10.1103/PhysRevB.13.2698

Publication date:

1976

\section{Document Version}

Publisher's PDF, also known as Version of record

Link back to DTU Orbit

\section{Citation (APA):}

Christensen, N. E. (1976). High-energy band structure of gold. Physical Review B, 13(6), 2698-2701. https://doi.org/10.1103/PhysRevB.13.2698

\section{General rights}

Copyright and moral rights for the publications made accessible in the public portal are retained by the authors and/or other copyright owners and it is a condition of accessing publications that users recognise and abide by the legal requirements associated with these rights.

- Users may download and print one copy of any publication from the public portal for the purpose of private study or research.

- You may not further distribute the material or use it for any profit-making activity or commercial gain

- You may freely distribute the URL identifying the publication in the public portal

If you believe that this document breaches copyright please contact us providing details, and we will remove access to the work immediately and investigate your claim 


\title{
High-energy band structure of gold
}

\author{
N. E. Christensen \\ Physics Laboratory I, The Technical University of Denmark, DK-2800 Lyngby, Denmark
}

(Received 15 July 1975)

\begin{abstract}
The band structure of gold for energies far above the Fermi level has been calculated using the relativistic augmented-plane-wave method. The calculated $f$-band edge $\left(\Gamma_{6}\right)$ lies $15.6 \mathrm{eV}$ above the Fermi level is agreement with recent photoemission work. The band model is applied to interpret temperaturemodulated reflectance data covering photon energies up to $35 \mathrm{eV}$.
\end{abstract}

Although the electronic energy-band structure of gold has been subject of many theoretical and experimental investigations, it is only recently that the energy range far above the Fermi level has been included in detailed examinations. The growing interest in the high-lying bands ( $f$ bands) is connected to the new experimental possibilities offered by synchrotron radiation facilities. In order to support the interpretation of optical experiments on gold where high photon energies are applied we present a relativistic augmented plane wave (RAPW) calculation that covers the highenergy regime. It is emphasized that the band calculation is based on the same potential as was used in previous studies ${ }^{1-3}$ of the bands in the neighborhood of the Fermi level, and therefore the actual position of the highly excited levels may not fit the experiments without adjustment. Although this reservation must be taken, it appears that the $f$-band edge is in agreement with experiments, ${ }^{4}$ and it will be demonstrated that it is possible from the present band structure to interpret the thermoreflectance data by Olson, Piacentini, and Lynch. ${ }^{5}$ These experiments cover photon energies up to $35 \mathrm{eV}$.

The energy bands have been calculated by means of the RAPW method as described earlier. ${ }^{1-3} \mathrm{Up}$ to $1.7 \mathrm{Ry}$ above the muffin-tin zero they are therefore almost identical to those used previously. In the extended calculation it has been necessary to use a dense $k$ mesh corresponding to 64 intervals on the $\Gamma-X$ line. The band structure at the main symmetry lines is shown ${ }^{6}$ in Fig. 1. The energies are given in rydbergs and measured from the muffin-tin zero. The Fermi level is $E_{F}=0.530 \mathrm{Ry}$ on this scale.

Modulation spectroscopy experiments are well suited $^{1}$ for comparison to band structure calculations since they emphasize critical-point transitions over the large noncritical background appearing in the static reflection traces. We shall here attempt an interpretation of the thermoreflectance data obtained by Olson, Piacentini, and Lynch. ${ }^{5}$ It will be assumed that the observed elements of structure can be related to Fermi-level transi- tions and transitions at critical points. Further, it is assumed that the most important critical points are to be found along the symmetry lines. Thus, only the part of the band structure which is shown in Fig. 1 will be considered.

The experimental values for spectral positions of critical-point thresholds were determined ${ }^{5}$ as the energies corresponding to the negative peaks ${ }^{7}$ in $\Delta R / R$. The first column of Table I gives the experimental ${ }^{5}$ edges for photon energies above 9 $\mathrm{eV}$. The second column contains critical-point energy separations as obtained in the present bandstructure calculation without any shifts. In many cases several critical-point transitions contribute to an element of structure, and only a detailed calculation of transition matrix elements can select those of most importance. The assignment presented here involves all the elements of structure in the experimental trace, ${ }^{5}$ whereas only nine were discussed by Olson, Piacentini, and Lynch. They related the observed spectral positions of structure elements to the band structure calculation by Connolly and Johnson. ${ }^{8}$ It was, however, necessary to introduce ${ }^{5}$ shifts in the computed bands of up to 1. $3 \mathrm{eV}$.

If the assignment suggested in Table $I$ is correct, then it follows that the experiment and the band calculation agree within $\sim 0.1 \mathrm{eV}$, as far as band separations are concerned. ${ }^{9}$ Further, it can be seen that our assignment differs somewhat from the one given in Ref. 5. The 10.0-eV transition is here assumed to take place at $W$ between band 3 and 6 (10.05 eV in the present calculation). The transition $3 \rightarrow 7$ at $K$ which was considered responsible in Ref. 5 is not a critical-point transition, and thus it is ruled out. The structure at $10.6 \mathrm{eV}$ appears to have contributions from the transition $4-8$ near $K$, but instead of $1 \rightarrow 7$ at $^{5} Q$ we suggest that the 4-8 transition at $W$ contributes. Concerning the structure at $13.2 \mathrm{eV}$ we agree with Ref. 5 that transitions from band 2 to 8 at $W$ can be responsible, but in addition the present band structure contains a critical point at the $\Sigma$ line $(2 \rightarrow 8)$. It cannot be expected that $2-8$ transitions ${ }^{5}$ at $K$ play any important role since there is no such 


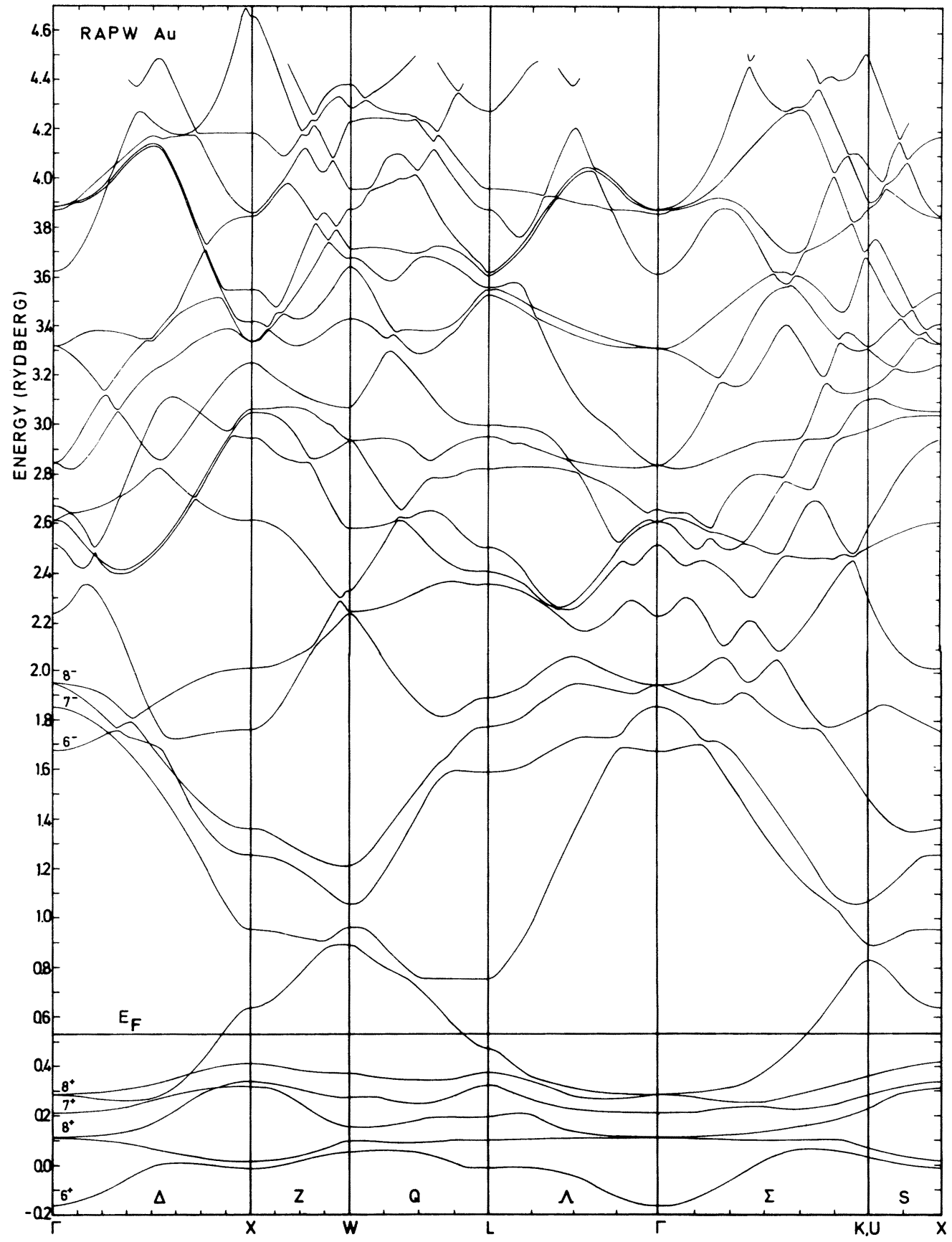

FIG. 1. Electronic energy bands for gold as calculated by the relativistic augmented plane wave method.

critical point (the considered bands are not parallel). The assignment of the element at $14.7 \mathrm{eV}$ represents the poorest agreement between the calculation and experiment. Tentatively it is suggested that transitions from the Fermi-level to band 8 , possibly in an extended region of $k$ space, contribute. The energy gap for such transitions $(6-8)$ is $14.54 \mathrm{eV}$ at $Q$. Olson, Piacentini, and Lynch did not assign any particular transitions to the 17.3-eV structure. Here it appears that two transitions both having band 8 as final state are involved, $1-8$ at $X$ and $4-8$ at $L$. Owing to the forbidden character of the $X$ transition it is expected that the $L$ transition dominates. The interpretation of the structure element at $19.9 \mathrm{eV}$ given in Table I differs essentially from the one by Olson et al., who ascribe it to a $\Gamma_{8}^{+} \rightarrow \Gamma_{7}^{-}$transition. It should be noted that this, in fact, must be re- 
TABLE I. Structure in the thermoreflectance spectrum of gold (Ref. 5) and the assignments to criticalpoint transitions in the calculated (RAPW) band structure (Fig。 1). The initial- and final-state band numbers are given in the parentheses. The levels are at each point numbered in order of increasing energy.

\begin{tabular}{|c|c|c|}
\hline $\begin{array}{l}\text { Experiment } \\
\text { (Ref. 5) (eV) }\end{array}$ & $\begin{array}{l}\text { Calculated energy } \\
\text { gap (eV) }\end{array}$ & Assignment \\
\hline 9.0 & 9.00 & $Q(2 \rightarrow 7)$ \\
\hline 10.0 & 10.05 & $W(3 \rightarrow 6)$ \\
\hline 10.6 & $\left\{\begin{array}{l}10.71 \\
10.71\end{array}\right.$ & $\begin{array}{l}W(4 \rightarrow 8) \\
K(4 \rightarrow 8)\end{array}$ \\
\hline 11.7 & 11.70 & $W(2 \rightarrow 7)$ \\
\hline 13.2 & $\left\{\begin{array}{l}13.10 \\
13.13\end{array}\right.$ & $\begin{array}{l}W(2 \rightarrow 8) \\
\Sigma(2 \rightarrow 8)\end{array}$ \\
\hline 14.7 & $\left\{\begin{array}{l}{[14.41} \\
14.54\end{array}\right.$ & $\begin{array}{l}W(3 \rightarrow 9)] \\
E_{F}(6) \rightarrow 8\end{array}$ \\
\hline 17.3 & $\left\{\begin{array}{l}17.31 \\
17.26\end{array}\right.$ & $\begin{array}{l}X(1 \rightarrow 8) \\
L(4 \rightarrow 8)\end{array}$ \\
\hline 19.9 & $\left\{\begin{array}{l}19.92 \\
19.84 \\
19.81\end{array}\right.$ & $\begin{array}{l}\Gamma(4 \rightarrow 7) \\
\Sigma(5 \rightarrow 10) \\
L(4 \rightarrow 9)\end{array}$ \\
\hline 21.2 & $\left\{\begin{array}{l}21.33 \\
21.23\end{array}\right.$ & $\begin{array}{l}\Gamma[(5,6) \rightarrow 8] \\
\Gamma[(2,3) \rightarrow 7]\end{array}$ \\
\hline 21.8 & $\left\{\begin{array}{l}21.65 \\
21.80\end{array}\right.$ & $\begin{array}{l}X(5 \rightarrow 11) \\
L(1 \rightarrow 8)\end{array}$ \\
\hline 22.7 & $\left\{\begin{array}{l}22.63 \\
22.75 \\
22.74 \\
22.79\end{array}\right.$ & $\begin{array}{l}\Gamma[(5,6) \rightarrow(9,10)] \\
\Sigma(2 \rightarrow 9) \\
X(4 \rightarrow 11) \\
L(2 \rightarrow 9)\end{array}$ \\
\hline 24.4 & $\left\{\begin{array}{l}24.20 \\
24.32 \\
24.43\end{array}\right.$ & $\begin{array}{l}\Sigma[(2,3) \rightarrow 9] \\
L(1 \rightarrow 9) \\
L(2 \rightarrow 10)\end{array}$ \\
\hline 27.7 & $\left\{\begin{array}{l}27.55 \\
27.47 \\
27.64\end{array}\right.$ & $\begin{array}{l}X(1 \rightarrow 11) \\
\Gamma(4 \rightarrow 11) \\
L(4 \rightarrow 11)\end{array}$ \\
\hline 28.6 & $\left\{\begin{array}{l}28.77 \\
28.71 \\
28.71\end{array}\right.$ & $\begin{array}{l}\Gamma[(2,3) \rightarrow 11] \\
\Sigma(2 \rightarrow 11) \\
\Sigma(5 \rightarrow 12)\end{array}$ \\
\hline 29.1 & $\left\{\begin{array}{l}29.10 \\
29.18 \\
29.00 \\
29.10\end{array}\right.$ & $\begin{array}{l}W(2 \rightarrow 10) \\
W(2 \rightarrow 11) \\
W(5 \rightarrow 13) \\
W(5 \rightarrow 14)\end{array}$ \\
\hline 31.0 & $\left\{\begin{array}{l}30.90 \\
(31.35)\end{array}\right.$ & $\begin{array}{l}X(4 \rightarrow 12) \\
{[\Gamma(4 \rightarrow 12)]}\end{array}$ \\
\hline 32.0 & $\left\{\begin{array}{l}32.18 \\
31.90 \\
32.10\end{array}\right.$ & $\begin{array}{l}L(1 \rightarrow 11) \\
L(6 \rightarrow 14) \\
L(6 \rightarrow 15)\end{array}$ \\
\hline
\end{tabular}

vised to $\Gamma_{8}^{+} \rightarrow \Gamma_{6}^{-}$since the $f$-band edge at $\Gamma$ is band 7 , which is a $\Gamma_{6}^{-}$level. The labeling used by Connolly and Johnson ${ }^{8}$ can not be correct at this point since $\Gamma_{7}^{-}$results from a spin-orbit splitting of the threefold degenerate level $\Gamma_{15}^{\prime}$ (single group notation). It follows from Table I that three critical points have the correct energy gap. The transition at $\Gamma(4-7)$ occurs between the $\Gamma_{7}^{+}$and $\Gamma_{6}^{-}$levels, and the initial-state energy $\left(\Gamma_{7}^{+}\right)$is 4. $34 \mathrm{eV}$ below the Fermi level. This value and the position of $\Gamma_{6}^{-}\left(15.58 \mathrm{eV}\right.$ above $\left.E_{F}\right)$ are both in excellent agreement with photoemission results obtained by Hermanson. Anderson, and Lapeyre, ${ }^{4}$ who synchronously varied the photon energy and the kinetic energy of the analyzed electrons to obtain "constant initial-state-energy spectra." It follows from the discussion above that also for the assignments given in Ref. 5 to the structure elements at 21.2 and $22.7 \mathrm{eV}$ alternative suggestions must be considered. The 21.2-eV element is here related to $\Gamma_{8}^{+} \rightarrow \Gamma_{7}^{-}$(in our labeling) and $\Gamma_{8}^{+} \rightarrow \Gamma_{6}^{-}$, whereas the $22.7-\mathrm{eV}$ structure may have four contributions, one being $\Gamma_{8}^{+} \rightarrow \Gamma_{8}^{-}$. Hermanson, Anderson and Lapeyre ${ }^{4}$ suggest that $\Gamma_{8}^{-}$[band (9, 10) at $\Gamma$ ] should be moved downwards ${ }^{6}$ by $0.8 \mathrm{eV}$ (from 19.32 to $18.55 \mathrm{eV}$ above $E_{F}$ ). This would not be consistent with the present interpretation of the thermoreflectance results, and further this would also imply that $\Gamma_{7}^{-}$(band 8 at $\Gamma$ ) should be displaced by the same amount since the separation of the levels $\Gamma_{8}^{-}, \Gamma_{7}^{-}$is determined by the spinorbit splitting. As in usual full-angle photoemission measurements on polycrystalline samples it cannot in general be expected that the peaks originate in localized regions in $k$ space. ${ }^{2}$ There are several bandpairs with $24.2-\mathrm{eV}$ separation and having the initial-state energy $-5.65 \mathrm{eV}$. The band diagram in Fig. 1 contains at least two such regions, $(2,3) \rightarrow 9$ at $\Lambda$ and $(2,3) \rightarrow 9$ at $\Sigma$, both in the neighborhood of $\Gamma$. In addition, the latter contains, in fact, a critical point. The negative peak in $\Delta R / R$ at $32 \mathrm{eV}$ has been related ${ }^{5}$ to the plasmon at $32.6 \mathrm{eV}$. The present calculation shows that in addition critical point transitions may contribute.

The present work demonstrates that it is possible to extend the band-structure calculation for gold far above the Fermi level and use the results in what seems to be a consistent interpretation of thermoreflectance experiments up to $35 \mathrm{eV}$. It is realized, however, that other band models may apply as well with other assignments. Therefore, only a careful calculation of transition probabilities and a line-shape analysis of the experimental trace can lend a more firm basis to the assignments. Apart from the comparison to the thermoreflectance experiment, we have presented a band calculation in the high-energy regime that may serve as a guideline for interpretations of future experiments using the synchrotron radiation. The necessity of such a calculation follows from observation ${ }^{5}$ that the optical spectra contain very sharp elements 
of structure even in this high-energy regime. This demonstrates the existence of well-defined energy bands far above the Fermi level, at least up to $33 \mathrm{eV}$; the plasmon energy referred to above.
${ }^{1}$ N. E. Christensen and B。O. Seraphin, Phys. Rev。B 4, 3321 (1971)。

${ }^{2}$ N. E. Christensen, Phys. Status Solidi B 52, 241 (1972)。

${ }^{3}$ N. E. Christensen, Solid State Commun. 949 (1971).

${ }^{4}$ J. Hermanson, J. Anderson, and G. Lapeyre, Phys. Rev. B 12,5410 (1975).

${ }^{5}$ C. G. Olson, M. Piacentini, and D. W. Lynch, Phys. Rev. Lett. 33, 644 (1974).

${ }^{6}$ The present calculation revealed that a plotting error appeared in the previous graphs (Refs. 1 and 2) at $\Delta$ for energies between 1.7 and 2.0 Ry since a $\Gamma_{7}^{-}$level erroneously was ascribed $\Gamma_{8}^{-}$symmetry.

${ }^{7}$ R. Rosei and D. W. Lynch, Phys. Rev。B $\underline{5}, 3883$ (1972).

${ }^{8}$ J. W. D. Connolly and K. H. Johnson, Massachusetts
Institute of Solid State and Molecular Theory Group Report No. 72,1970 , p. 19 (unpublished).

${ }^{9}$ The comparison between calculated energies of individual levels should be done with some caution, since the RAPW calculation was performed using the Hetemperature lattice constant (Ref. 1), whereas most optical experiments are carried out at room temperature. We have performed RAPW calculations over the entire Brillouin zone for three different lattice constants, showing that, the majority of the levels are shifted downwards by $0.15-0.22 \mathrm{eV}$ relative to $E_{F}$, if the $300-\mathrm{K}$ lattice constant is used instead of the $4.2-\mathrm{K}$ value. This implies that the comparison in Table $\mathrm{I}$ is not affected since the thermoreflectance experiment was carried out at $200 \mathrm{~K}$. 